\title{
Prevalencia puntual del trastorno de estrés postraumático y su relación con indicadores altos de burnout en personas del sector justicia de Guatemala
}

\author{
Point prevalence of post-traumatic stress disorder and its relationship with high indicators of \\ burnout in people of the justice sector from Guatemala \\ Daniel E. Sojuel, Llomauri Cifuentes-Guerra, Walter O. Paniagua* \\ Centro de Investigaciones en Psicología “Mayra Gutiérrez”, Escuela de Ciencias Psicológicas, \\ Universidad de San Carlos de Guatemala, Guatemala. \\ *Autor al que se dirige la correspondencia: paniagua.omar@usac.edu.gt
}

Recibido: 14 de enero 2017 / Revisión: 18 de abril 2017 / Aceptado: 02 de junio 2017

\section{Resumen}

Q scasos estudios se han realizado en Guatemala con profesionales del sector justicia que trabajan con víctimas de delitos, caracterizados por estar expuestos a sucesos que pueden considerarse como traumáticos. El objetivo del estudio fue determinar la prevalencia puntual del trastorno de estrés postraumático (TEPT) en una institución del sector justicia de Guatemala. Se realizó una triangulación de datos cuantitativos, obtenidos mediante la escala de estrés traumático secundario (STSS) y cualitativos, obtenidos a través de las respuestas a una pregunta adicional de sucesos traumáticos. Como resultado, la prevalencia puntual de estrés postraumático fue de $15 \%$, en donde el síntoma de TEPT más frecuente fue el de intrusión. Además, se evaluó la presencia del síndrome burnout a través de una subescala de la escala de calidad de vida profesional (ProQOL v.5). Los resultaron mostraron un 62\% de prevalencia puntual de indicadores significativos de burnout; y como factor de riesgo asociado a TEPT, se obtuvo una razón de probabilidad de 5.93, con intervalo de confianza de 95\% $[2.47,14.27](p<.001)$. Finalmente, la presencia de sintomatología de TEPT fue contrastada con aquellos eventos indicados como traumáticos por los sujetos de investigación. Se determinó que las situaciones predisponentes al desarrollo de los síntomas de TETP, además de los indicados en el Manual de Diagnóstico y Estadístico de los Trastornos Mentales en su quinta revisión (DSM-5), se relacionan con aspectos administrativos como la sobrecarga laboral. Estos hallazgos pueden contribuir al desarrollo de programas de autocuidado y bienestar psicológico basados en evidencia empírica.

Palabras claves: Salud mental, trauma psicológico, factores de riesgo, triangulación de información, estrés laboral

\section{Abstract}

$\mathrm{F}$ ew studies have been conducted in Guatemala with professionals in the justice sector who work with victims of crime, characterized by being exposed to events that can be considered traumatic. The objective of this study was to determine the point prevalence of post-traumatic stress disorder (PTSD) in a justice institution in Guatemala. Triangulation was performed with quantitative data, resulting from the scores of the secondary traumatic stress scale (STSS) and qualitative, obtained from the responses to an additional question of traumatic events. As a result, the point prevalence of posttraumatic stress was $15 \%$, where the most frequent PTSD symptom was intrusion. In addition, the presence of burnout syndrome was evaluated through a subscale of the professional quality of life scale (ProQOL v.5). The results showed a 62\% prevalence of significant indicators of burnout; And as a risk factor associated with PTSD, an odds ratio of 5.93 was obtained, with a 95\% confidence interval $[2.47,14.27](\mathrm{p}<.001)$. Finally, the presence of PTSD symptomatology was contrasted with those events indicated as traumatic by the research subjects. It was determined that the conditions predisposing to the development of TETP symptoms, in addition to those indicated in the Diagnostic and Statistical Manual of Mental Disorders in its fifth review (DSM5), are related to administrative aspects such as work overload. These findings may contribute to the development of self-care and psychological well-being programs based on empirical evidence. 


\section{Introducción}

Los altos índices de violencia en Guatemala permiten estudiar las consecuencias psicológicas del estrés debido a que facilitan el escenario para la génesis de un trauma, que es la causa necesaria para el despliegue del trastorno de estrés postraumático (TEPT) y una causa contributiva en el desarrollo de otras psicopatologías (Dunn, Nishimi, Powers, \& Bradley, 2017).

Radke-Yarrow y Sherman (1992) argumentan que el desarrollo de síntomas a eventos potencialmente traumáticos está condicionado por la variabilidad de la respuesta humana, la cual parte de capacidades cognitivas y emocionales para adaptarse a condiciones de emergencia.

El estrés que se produce luego del acontecimiento, hace del trauma un fenómeno que se desarrolla secuencialmente porque implica una ejecución inadecuada de estrategias de afrontamiento al experimentar situaciones problemáticas (Prati \& Pietrantoni, 2009).

Si las vivencias estresantes posteriores al acontecimiento ocurren por más de un mes, el trauma se consolida a partir de vivencias emocionales poco adaptativas, distorsión de esquemas cognitivos, evitación de estímulos y reexperimentación, lo que configura un cuadro de TEPT (American Psychological Association [APA], 2014).

En detalle, la diagnosis de TEPT apunta a una triada sintomática relacionada con presencia de intrusión, evitación e hiperalerta; además, las dimensiones de TEPT se asocian a dos aspectos distintivos ya señalados: una respuesta patológica al estrés y la presencia o desarrollo de trauma. La reexperimentación se presenta por procesos oníricos, síntomas somáticos y emocionales, además de malestar psicológico y modificación de la identidad personal, las concepciones del mundo y de los otros; finalmente, se despliega un estado de hiperalerta que conlleva a irritación y respuestas de sobresalto (APA, 2014).

Se debe reconocer un punto esencial respecto a la dinámica traumática desde el contexto donde se produce. Se han determinado asociaciones entre TEPT y la exposición a eventos traumáticos en el trabajo de intervención con personas expuestas a situaciones de violencia y emergencia (Lima \& Assunção, 2011).

En adición, Arón y Llanos (2004) establecen que operadores sociales que trabajan con víctimas directas de hechos violentos pueden desarrollar sintomatología asociada a burnout. La justificación más básica que se realiza para indicar burnout y no otro trastorno cuan- do se hace referencia a trabajo con víctimas directas de hechos violentos, se relaciona con la intención de señalar que la causa u origen del problema psicológico se encuentra en el ámbito laboral para evitar colocar el foco problemático en las características del profesional.

Sin embargo, al trabajar de forma recurrente con situaciones de alto impacto emocional, se pueden producir efectos que no necesariamente son explicados desde las condiciones de trabajo. Las problemáticas como contaminación temática, traumatización vicaria, desgaste por empatía, entre otros, pueden ser mejor entendidas desde la categoría de estrés traumático secundario (Figley, 1995).

Se ha reportado relación entre burnout y estrés traumático secundario en estudios con trabajadores expuestos indirectamente a procesos traumáticos (Cieslak et al., 2014). La prueba utilizada para evaluar estas variables es la escala de calidad de vida profesional, en su quinta versión (ProQOL v.5) desarrollada por Stamm (2010) y se caracteriza por dos aspectos, uno positivo y el otro negativo.

$\mathrm{Al}$ aspecto positivo se le nombró satisfacción por compasión, y se relaciona con el placer derivado de realizar adecuadamente el trabajo; por ejemplo, la satisfacción encontrada al ayudar a otras personas. Al aspecto negativo se le llamó fatiga por compasión, que se refiere al agotamiento emocional y físico que se desarrolla en profesionales de ayuda (Figley, 1995).

Adams, Boscarino y Figley (2006) llegaron a la conclusión que el burnout y el estrés traumático secundario eran dos componentes de la fatiga por compasión. En este proceso, Stamm (2010), desarrolla la escala ProQOL, la cual utiliza el concepto de fatiga por compasión y la satisfacción por compasión, ambos relacionados con la calidad de vida laboral.

El burnout, en este caso, está asociado con el sentimiento de desesperanza y dificultad de lidiar con el trabajo o para realizarlo de manera efectiva, y las manifestaciones pueden tener un desarrollo gradual. En cambio, el estrés traumático secundario como segundo componente de la fatiga por compasión, se entiende como un efecto de la exposición indirecta a eventos traumáticos o estresantes (Stamm, 2010).

Una consideración importante sobre el estrés traumático secundario es que los síntomas presentados en este cuadro son similares a los presentados por TEPT (Chrestman, 1995; Figley, 1999). La diferencia entre ambos corresponde al tipo de exposición al acontecimiento traumático, es decir, si los parámetros del criterio A para TEPT del DSM-5 corresponden a exposición 
directa de eventos amenazantes contra la vida (criterio A1 y criterio A2), o bien, por exposición indirecta debido a detalles del suceso (criterios A3 y A4).

En la investigación realizada por Bride (2007), se asume al estrés traumático secundario como una consecuencia directa de la atención a víctimas de hechos violentos. Los resultados demuestran que el $29 \%$ de los trabajadores de temática social presentaban síntomas cognitivos de intrusión de forma frecuente, $10 \%$ evitaba personas, lugares o cosas relacionados con los detalles de eventos traumáticos y $21 \%$ presentaba disminución del nivel de actividad; además, $22 \%$ de los participantes manifiestan irritabilidad y $10 \%$ hipervigilancia de manera frecuente.

Follette, Polusny y Milbeck (1994), en un estudio descriptivo con profesionales de la ley y profesionales de la salud mental expuestos a material traumático de abuso sexual infantil, concluyeron que los profesionales de la salud mental evaluados reportaron niveles bajos de síntomas traumáticos, distrés y moderado nivel de estrés personal, mientras que los profesionales de la ley presentaron niveles altos de estas mismas variables.

Otras investigaciones (McElroy \& McElroy, 1991; McCann \& Pearlman, 1990), manifiestan que la identificación con relatos de sucesos traumáticos debido a experiencias personales similares puede ser un importante precursor para el aparecimiento de síntomas de TEPT.

La recapitulación de estos estudios hace especial hincapié en la traumatización secundaria asociada a la atención de víctimas de hechos traumáticos; sin embargo, debe considerarse con el DSM-5, que la instauración de TEPT también puede generarse de forma indirecta.

De esta forma, la investigación se orientó a determinar cuál era la prevalencia de TEPT en personas que trabajan en una institución del sector justicia en la ciudad de Guatemala durante el año 2016, establecer qué sintomatología era más recurrente (reexperimentación, evitación y embotamiento afectivo o hiperactivación); identificar qué sucesos son considerados como traumáticos; y determinar si los indicadores significativos de burnout son factores de riesgo para el desarrollo del TEPT.

\section{Materiales y métodos}

\section{Método de recolección de datos}

La investigación se desarrolló con 366 trabajadores que representan $91 \%$ del total de la población de tres departamentos de una de las instancias del sector justicia de Guatemala, siendo el criterio de inclusión, laborar en una de estas tres áreas. Se recolectó la información durante un mes y se obtuvo un porcentaje de omisión censal de $9 \%$, conformado por personas que decidieron no participar en el estudio, o por no poder asistir en los días u horarios en las que se realizaron las evaluaciones.

\section{Instrumentos y procedimiento}

Como parte del proceso de investigación, se realizó un plan piloto con 62 miembros de la población. A partir de este plan, se adaptó el contenido de las escalas STSS y ProQOL-v5 al contexto y se agregaron aspectos importantes para una evaluación más rigurosa.

Para establecer la prevalencia de TEPT se utilizó la escala STSS desarrollada por Bride, Robinson, Yegidis y Figley (2004), más una pregunta adicional elaborada por el equipo de investigación que tuvo como objetivo evitar el sobrediagnóstico.

La pregunta adicional se presentó de la siguiente forma: "Piense en una situación o evento del trabajo que ocurrió en los últimos 30 días y que le haya generado horror, temor o desesperanza. Intente narrar la situación o el evento expresando libremente sus impresiones e ideas. Tome el tiempo necesario para elaborar la descripción. No son importantes la ortografía, ni la gramática o la sintaxis". A través de las respuestas, se indagó sobre la presencia del criterio A del DSM-5 $\mathrm{y}$ otras reacciones personales no contempladas como criterios diagnósticos de TEPT en el DSM-5.

La elaboración de la pregunta adicional parte de la importancia de los procesos emocionales como respuesta a la exposición a sucesos traumáticos, aspecto que se rescata del DSM VI-TR y que en esta investigación se utilizó, no como categoría de análisis, sino como herramienta evocadora de respuestas que serían categorizadas dentro de los criterios del DSM-5.

Como complemento, para determinar la existencia de indicadores de compasión por satisfacción y burnout se aplicó la escala ProQOL-v.5, creada por Stamm (2010). Se pidió a los participantes leer cada ítem de las escalas e indicar la frecuencia en que estos 
se adecuan a sus vidas durante el último mes (temporalidad fundamentada en el DSM-5), utilizando una escala Likert de cero (nunca) a cuatro (muy frecuente); y en el caso de la escala ProQOL-v.5, el rango fue de cero (nunca) a cinco (muy seguido), criterios apegados a las adaptaciones originales.

\section{Análisis estadístico}

La escala STSS consta de 17 ítems y se compone de tres subescalas: intrusión (ítems: 2, 3, 6, 10, 13), evitación (ítems: 1, 5, 7, 9, 12, 14, 17) e hiperactivación (ítems: 4, 8, 11, 15, 16). La presencia del TEPT se determinó mediante un algoritmo, compuesto como mínimo por un indicador de intrusión, tres indicadores de evitación y dos indicadores de hiperactivación para establecer la presencia del TEPT.

Para la evaluación de la compasión por satisfacción, burnout y estrés traumático secundario, se utilizó la escala ProQOL-v.5, la cual incluyó 30 ítems, 10 para cada una de sus tres subescalas (compasión por satisfacción, burnout y estrés traumático secundario).

La forma de interpretar los resultados fue mediante las tres subescalas separadas, para ello, el punto de corte establecido en la quinta revisión por Stamm (2010) fue un puntaje bruto de 23. Es importante resaltar que los resultados de esta escala no son diagnósticos, más bien son alertas para procesos evaluativos clínicos más rigurosos.

Para la escala de burnout se invirtieron los puntajes de los ítems formulados en positivo $(3,14,16,19 \mathrm{y}$ 29) para favorecer la sumatoria de la escala. Por tanto, un punteo mayor o igual a 23 en la subescala de burnout (items: 3 invertido, 7, 9, 14 invertido, 16 invertido, 18, 19 invertido, 21, 26 y 29 invertido) y en la de estrés traumático secundario (ítems: 1, 4, 6, 8, 10, 12, 13, 23, 25 y 28) representará niveles significativos de estos dos procesos en las personas evaluadas.

Por otro lado, un puntaje igual o mayor a 23 en la subescala de compasión por satisfacción (ítems: 2 , $5,11,15,17,20,22,24,27$ y 30 ) indica que la persona evaluada experimenta satisfacción significativa con su habilidad de trabajar con otras personas.

En adición, se determinó la confiabilidad a partir del coeficiente alfa de Cronbach, aplicada a cada una de las subescalas de ambas escalas antes descritas, se evaluó la validez de constructo a partir del análisis factorial exploratorio.

Por último, se compararon aspectos demográficos y la presencia o ausencia del TEPT mediante la prueba ji cuadrado y se realizó un análisis de razón de proba- bilidad, para determinar si el burnout es un factor de riesgo para el desarrollo del TEPT.

\section{Aspectos éticos}

La investigación fue revisada por el Comité de Bioética en Investigación en Salud de la Universidad de San Carlos de Guatemala. Por otro lado, se informó a los participantes que la evaluación no conllevaba riesgos para la salud física o psíquica y que en cualquier momento se podía dejar de participar en el estudio.

Las escalas se aplicaron con grupos establecidos de participantes durante un mes, se mantuvo la confidencialidad de los participantes a través de un código personal para identificar sus resultados al momento de la devolución y retroalimentación de estos.

Posterior a la evaluación, se devolvieron los resultados a los participantes de manera individual durante tres semanas; se realizaron recomendaciones pertinentes para cada caso de forma verbal, resolviendo dudas y se orientó para realizar acciones de prevención y atención de la salud mental. Se obtuvo un porcentaje de participación del 26\% para la devolución.

\section{Resultados}

La población estuvo conformada por 53\% mujeres, $45 \%$ hombres y $7 \%$ que no especificó el sexo; el promedio de edad fue de 36 años ( $\mathrm{SD}=9.6)$; en cuanto al estado civil, $48.9 \%$ fueron personas casadas o en unión libre, $43.1 \%$ de la población fue soltera y el $6 \%$ divorciada o separada. Otros aspectos demográficos laborales relacionados con el trabajo fueron: la asignación de turnos nocturnos, en donde se observó un $27 \%$; además, el $96 \%$ de las personas refirió que asiste al trabajo estando enfermos.

Para la confiabilidad de la escala STSS, se calculó el coeficiente alfa de Cronbach, del cual se observan los siguientes resultados para cada una de las subescalas y la escala total: intrusión $(\alpha=.8)$, evitación $(\alpha=.8)$, hiperactivación $(\alpha=.8)$ y escala total $(\alpha=.9)$. Para la validez de constructo, se realizó un análisis factorial exploratorio. La prueba Kaiser Meyer Olkin (KMO) brindó un resultado de $\mathrm{KMO}=.9$ y la prueba de esfericidad de Bartlett indicó que existen correlaciones significativas entre las variables $(p<.05)$. Para la confiabilidad de la escala ProQOL-v.5 de calidad de vida profesional, el coeficiente alfa de Cronbach mostró los siguientes resultados: subescala de compasión por satisfacción $(\alpha=9)$, burnout $(\alpha=.7)$ y estrés traumático secundario $(\alpha=.8)$. 
Con respecto a la validez de constructo, la prueba KMO dio como resultado .9 y la prueba de esfericidad de Bartlett muestra correlaciones significativas entre las variables $(p<.05)$.

A partir de la escala STSS y el análisis cualitativo de la pregunta adicional, se estableció una prevalencia puntual de $15 \%$. Se puede observar en la Tabla 1, la prevalencia desagregada por aspectos demográficos. Se determinó mediante la prueba de ji cuadrado, que existe diferencia significativa entre la prevalencia del TEPT, entre mujeres y hombres $(p<.05)$.

En cuanto a la presencia de los indicadores evaluados en las subescalas, se sumó el resultado de cada una de estas y se compararon los porcentajes para cada una (intrusión $=1.5$, hiperactivación $=1.5$ y evitación $=$ 1.1). Por tanto, se afirma que el indicador más frecuente en la población total es el de intrusión, seguido por el de hiperactivación y, por último, la evitación. Para los sucesos traumáticos identificados en el análisis de la pregunta adicional, se encontró que el 9.6\% de las personas evaluadas han experimentado directamente un suceso traumático, en adición, según las referencias codificadas, se encuentran 35 tipos de eventos traumáticos.

La manifestación traumática directa incluye la exposición a asaltos, la realización de atribuciones laborales en lugares considerados como zonas rojas, amenazas de muerte, peligro inminente para la familia,

Tabla 1

Prevalencia de TETP, desagregado por aspectos demográficos

\begin{tabular}{|c|c|c|c|c|}
\hline \multirow[b]{2}{*}{ Caracterización de la población } & \multicolumn{2}{|c|}{ Presencia de TEPT } & \multicolumn{2}{|c|}{ Ausencia de TEPT } \\
\hline & Frecuencia & $\%$ & Frecuencia & $\%$ \\
\hline \multicolumn{5}{|l|}{ Sexo } \\
\hline Hombres & 18 & 32.7 & 147 & 47.3 \\
\hline Mujeres & 35 & 63.6 & 159 & 51.1 \\
\hline No identificados & 2 & 3.6 & 5 & 1.6 \\
\hline \multicolumn{5}{|l|}{ Estado civil } \\
\hline Soltero & 29 & 52.7 & 129 & 41.5 \\
\hline Casado/Unión libre & 23 & 41.8 & 156 & 50.2 \\
\hline Separado & 0 & 0 & 13 & 4.2 \\
\hline Divorciado & 1 & 1.8 & 8 & 2.6 \\
\hline Perdidos & 2 & 3.6 & 5 & 1.6 \\
\hline \multicolumn{5}{|l|}{ Nivel de formación } \\
\hline Licenciatura & 28 & 50.9 & 141 & 45.3 \\
\hline Bachillerato & 15 & 27.3 & 81 & 26 \\
\hline Maestría & 6 & 10.9 & 38 & 12.2 \\
\hline Técnico & 4 & 7.3 & 35 & 11.3 \\
\hline Doctorado & 0 & 0 & 3 & 1 \\
\hline Perdidos & 2 & 3.6 & 13 & 4.2 \\
\hline \multicolumn{5}{|l|}{ Realización de turnos } \\
\hline No & 32 & 58.2 & 235 & 75.6 \\
\hline Sí & 23 & 41.8 & 76 & 24.4 \\
\hline \multicolumn{5}{|l|}{ Hijos } \\
\hline No & 27 & 49.1 & 117 & 37.6 \\
\hline Sí & 25 & 45.5 & 170 & 54.7 \\
\hline
\end{tabular}


e intimidaciones. Estos resultados constituyen sucesos traumáticos descritos dentro del criterio A de TEPT, los cuales pueden observarse en la Tabla 2. El análisis también tomó en cuenta eventos que no pueden conceptualizarse sobre los criterios del TEPT, pero cuya aparición fue considerada relevante para la población estudiada. En la Tabla 3 se observan estos eventos.

Para la escala ProQOL-v.5, a partir de los puntos de corte de cada subescala se puede identificar si los indicadores son significativos, por tanto, la prevalencia de personas con indicadores significativos de satisfacción por compasión es de $94 \%$; la prevalencia de personas con indicadores significativos de burnout es de 62\%; y, por último, la prevalencia de personas con indicadores significativos de exposición estrés traumático secundario es de $87 \%$. Estos indicadores no representan un diagnóstico clínico, son más bien una alerta para prevención de futuras afectaciones.

$\mathrm{Al}$ analizar los resultados mediante el cálculo de razón de probabilidad entre la prevalencia del TEPT y la subescala de burnout de la escala ProQOL-v.5, se obtiene un resultado de 5.9, con intervalo de confianza del 95\% [2.47, 14.27] $(p<.001)$.

\section{Discusión}

Para la adaptación realizada en la población evaluada de la escala STSS y la escala ProQOL-v.5, los resultados de la prueba de confiabilidad alfa de Cronbach indicaron pertinencia y adecuación de los ítems ante la medición de los constructos de ambas escalas, por lo que se afirma que los instrumentos utilizados son confiables. En cuanto a la validez de constructo, mediante la prueba KMO, se concluyó que los ítems analizados, para ambas escalas, están correlacionados entre sí y se pueden realizar extracciones; además, la prueba de esfericidad de Bartlett indicó que existe ajuste de los datos al análisis factorial exploratorio, lo que indica una adecuada validez de constructo.

Para la escala STSS, no se realizó extracción de ítems, debido a que el análisis se apegó al algoritmo de la escala original para establecer la prevalencia, pero se observa que el ítem 1 "Me sentí emocionalmente entumecido" puede ser un ítem extraíble de la escala, ya que su carga factorial fue de $<.4$. Se interpreta este resultado debido a la traducción insuficiente del ítem a partir de la adaptación original.

Son escasos los estudios realizados con personas que atienden a víctimas de violencia común; sin embargo, Carvajal (2002) reportó prevalencia en distin- tos tipos de muestras de norteamericanas y refiere que, en Estados Unidos, para personas testigos de muertes graves o lesiones, la prevalencia del TEPT es de $11.9 \%$ y para personas que se han informado de hechos traumáticos es de $4.6 \%$. En una muestra argentina de distintos profesionales de salud que han participado en atención con víctimas múltiples, en distintos eventos, como accidentes o desastres naturales, se reportó una prevalencia de $8.9 \%$ (Abaz, Babbino, Volpi, Orlando, \& Valdez, 2016).

En el presente estudio, se indica una prevalencia del $15 \%$, porcentaje que necesita ser comparado con otras poblaciones o muestras en Guatemala. Es necesario resaltar que las diferencias de género en el desarrollo de TEPT, son comunes para los diferentes tipos de grupos (Blanco \& Amarís, 2014; Carvajal, 2002), en este estudio también se encontraron diferencias significativas, en donde las mujeres tienen una mayor prevalencia de TEPT.

A pesar de estipularse que las personas que trabajan dentro del sector justicia son una población vulnerable al desarrollo del TEPT debido a la exposición continuada de detalles repulsivos, también se enmarcan factores de riesgo asociados al trabajo que corresponden a una franca exposición de peligro real.

Aunque la población evaluada especificó el tipo de evento traumático por o durante un mes, según las comparaciones realizadas entre situaciones traumáticas correspondientes al criterio diagnóstico A y sintomatología TEPT, no todas las personas expuestas desarrollaron los síntomas, potenciando una posible correlación con el tipo de respuesta adaptativa, características de personalidad y aspectos situacionales de la vida personal (Loo et al., 2016).

Gibbons, Murphy y Joseph (2011) prueban en su investigación que los síntomas asociados al TEPT pueden presentarse en aquellos profesionales que desarrollan contratransferencia con las víctimas de hechos traumáticos que atienden. Es decir, tienden a identificarse con las vivencias personales de esos casos debido a experiencias similares. Esto permite dilucidar otro factor de riesgo para las personas que trabajan con hechos delictivos según el estudio de Wrenn (2005), pues pone de manifiesto que la necesidad de ayudar y el experimentar circunstancias parecidas a las de las víctimas, representa un riesgo para el desarrollo del TEPT.

En aquellos casos en los que el evento traumático se estructura únicamente a partir de detalles repulsivos, el estudio establece que el $23.5 \%$ indicó haber sido expuesto de forma indirecta a un hecho impactante. Por 
Tabla 2

Sucesos traumáticos asociados al criterio diagnóstico A de TEPT del DSM 5

\begin{tabular}{|c|c|c|c|c|}
\hline \multirow[b]{2}{*}{ Criterios diagnósticos } & \multicolumn{2}{|c|}{ Casos } & \multicolumn{2}{|c|}{ Referencias } \\
\hline & Frecuencia & $\begin{array}{l}\text { \% según } \\
n\end{array}$ & $\begin{array}{l}\text { Referencias codi- } \\
\quad \text { ficadas }\end{array}$ & $\%$ \\
\hline \multicolumn{5}{|l|}{ A1 } \\
\hline Experiencia directa de sucesos traumáticos & 35 & 9.6 & 35 & 28 \\
\hline \multicolumn{5}{|l|}{$\mathrm{A} 2$} \\
\hline Presencia directa del suceso ocurrido a otros & 3 & .8 & 3 & 2.4 \\
\hline \multicolumn{5}{|l|}{ A 3} \\
\hline $\begin{array}{l}\text { Conocimiento de que el suceso ha ocurrido a un amigo } \\
\text { íntimo. En casos de amenaza o realidad de muerte de un } \\
\text { familiar o amigo, el suceso debió ser violento o accidental }\end{array}$ & 1 & .3 & 1 & .8 \\
\hline \multicolumn{5}{|l|}{ A4 } \\
\hline $\begin{array}{l}\text { Exposición repetida o extrema a detalles repulsivos del } \\
\text { suceso traumático }\end{array}$ & 82 & 22.4 & 86 & 68.8 \\
\hline Total & 121 & 33.1 & 125 & 100 \\
\hline
\end{tabular}

Tabla 3

Sucesos no asociados a criterios diagnósticos de TEPT del DSM 5

\begin{tabular}{|c|c|c|c|c|}
\hline \multirow[b]{2}{*}{ Sucesos } & \multicolumn{2}{|c|}{ Casos } & \multicolumn{2}{|c|}{ Referencias } \\
\hline & Frecuencia & $\begin{array}{l}\text { \% según } \\
n\end{array}$ & Frecuencia & $\%$ \\
\hline Aspectos administrativos & 49 & 13.4 & 49 & 34.5 \\
\hline $\begin{array}{l}\text { Aspectos relacionados a atención de casos: impotencia y } \\
\text { relaciones ineficientes }\end{array}$ & 14 & 3.8 & 14 & 9.9 \\
\hline Insatisfacción laboral & 8 & 2.2 & 8 & 5.6 \\
\hline Circunstancias personales: duelo & 3 & .8 & 3 & 2.1 \\
\hline Ineficacia laboral & 20 & 5.5 & 20 & 14.1 \\
\hline Co-relaciones laborales insatisfactorias & 7 & 1.9 & 7 & 4.9 \\
\hline Sobrecarga laboral & 28 & 7.7 & 29 & 20.4 \\
\hline Liderazgo negativo & 11 & 3.0 & 12 & 8.5 \\
\hline Total & 140 & 38.3 & 142 & 100 \\
\hline
\end{tabular}


ello, se considera que el trauma secundario es un factor concomitante que se relaciona con la ayuda a víctimas primarias de hechos traumáticos.

Se comprende el estrés traumático secundario como una forma de TEPT (Bride et al., 2004), aún existen algunos reparos que son necesarios realizar; como lo define Horesh (2015), la sola exposición indirecta a un evento traumático como es definido por el criterio A, no es condición suficiente para desarrollar los síntomas del TEPT. La investigación empírica ha definido que es necesario considerar la historia traumática, las habilidades emocionales, el nivel de identificación con el suceso, empatía, estilos de afrontamiento y características de personalidad para definir completamente un cuadro secundario de TEPT, es decir, un diagnóstico sostenido por los criterios A3 y A4 del DSM-5.

Horesh (2015) también establece que existen ciertas implicaciones que surgen de la asimilación del estrés traumático secundario al diagnóstico de TEPT en el DSM-5, puesto que no se consideran otras variables. De forma particular, podemos establecer que los resultados cualitativos de la investigación apuntan a situaciones consideradas traumáticas por los participantes, pero que no son sustentadas por los criterios diagnósticos para TEPT; esto implica que deben ser situaciones explicadas por otro tipo de cuadro clínico como la presencia de burnout; y, como se detalló, existe una presencia significativa de estrés laboral en la población de estudio.

Se visualiza una nueva dimensión sobre la consideración del trauma para trabajadores del sector justicia, debido a que aspectos administrativos, insatisfacción laboral, interrelaciones laborales insatisfactorias, sobrecarga laboral continua y liderazgo negativo pueden ser condiciones predisponentes para el surgimiento de un trauma, cuyas manifestaciones podrían ser similares al diagnóstico de TEPT o emerger con una sintomatología distintiva.

Debido a la variabilidad de los resultados respecto a los sucesos traumáticos, se estableció la necesidad de observar la situación desde otras perspectivas, en este caso, a través de los niveles significativos de burnout. Existen estudios donde al correlacionar burnout con TEPT, la relación no es evidente, por ejemplo, Katsavouni, Bebetsos, Malliou y Beneka (2016), con una muestra de bomberos, no se encontró correlación clara. Por otro lado, la correlación en un estudio con muestra de personal de una correccional fue alta (Boudoukha, Altintas, Rusinek, Fantini-Hauwel, \& Hautekeete, 2013). Estas diferencias suponen que las características personales de la población estudio pueden afectar los resultados de las correlaciones.
Para determinar si los niveles de burnout en la población son un factor de riesgo, se utilizó el cálculo de razón de probabilidad por su utilidad en el campo (Glas, Lijmer, Prins, Bonsel, \& Bossuyt, 2003). En otros estudios se ha considerado la exposición a una situación o una característica demográfica de la muestra y se ha comparado con la presencia o ausencia de TEPT (Breslau, Davis, \& Anreski 1995; Cuffe et al., 1998; Meadors, Lamson, Swanson, White, \& Sira, 2010).

La diferencia de este estudio con los citados, es que se utilizaron los resultados de la subescala de burnout de la prueba ProQOL v.5, tomando los casos con niveles significativos de burnout y los casos no significativos se comparan con la prevalencia de TEPT, se concluye que los indicadores significativos de burnout son un factor de riesgo para el desarrollo de TEPT. Es importante resaltar que conocer los factores de riesgo, permite guiar acciones necesarias para la prevención del TEPT en personal de ayuda.

Los hallazgos cubrieron los objetivos de la investigación solo en cierta medida. Debido a la organización institucional, el estudio se realizó en el distrito de la ciudad capital, lo que limita el alcance de la investigación; por ello, dos distritos con índices elevados de violencia no pudieron ser considerados para el estudio. A pesar de haberse determinado la prevalencia para el diagnóstico de TEPT, se disminuyeron las líneas de discusión importantes que pudieran comparar la situación de violencia, al considerar que personas que trabajan dentro del sector justicia, también pueden ser víctimas primarias de hechos delictivos o violentos. Esta observación y la posibilidad de comparación pueden abrir parámetros para comprender la instauración y evolución del diagnóstico en las dependencias contra el delito.

También es relevante mencionar que la investigación arroja datos para la creación de programas de autocuidado, o desarrollo de bienestar psicológico, basados en el entendimiento de las problemáticas que afectan al personal del sector justicia. En contextos altamente violentos como el guatemalteco, la humanización del trabajo es un tema primordial para el desarrollo y cuidado de la salud mental.

\section{Agradecimientos}

Esta investigación fue cofinanciada por la Dirección General de Investigación de la Usac durante el año 2016, con partida presupuestaria número 4.8.63.1.83. 


\section{Referencias}

Abaz, B., Babbino, V., Volpi, M., Orlando, G., \& Valdez, P. (2016). Estudio del estrés postraumático en personal de salud que ha participado en eventos con víctimas múltiples. Revista Argentina de Medicina, 4(9), 40-49.

Adams, R. E., Boscarino, J. A., \& Figley, C. (2006). Compassion fatigue and psychological distress among social workers: A validation study. American Journal of Orthopsychiatry, 76(1), 103108. doi: 10.1037/0002-9432.76.1.103

American Psychological Association. (2014). Diagnostic and Statistical Manual of Mental Disorders (DSM-5). Madrid: Editorial Médica Panamericana.

Arón, A., \& Llanos, M. (2004). Cuidar a los que cuidan: Desgaste profesional y cuidado de los equipos que trabajan con violencia. Sistemas Familiares, 20(1-2), 5-15.

Blanco, A., \& Amarís, M. (2014). La ruta psicosocial del desplazamiento: Una perspectiva de género. Universitas Psychologica, 13(2), 661-679. doi. org/10.11144/Javeriana.UPSY13-2.rpdu

Boudoukha, A. H., Altintas, E., Rusinek, S., Fantini-Hauwel, C., \& Hautekeete, M. (2013). Inmates to staff assaults, PTSD and burnout: Profiles of risk and vulnerability. Journal of Interpersonal Violence, 28(11), 2332-2350. doi: 10.1177/0886260512475314

Breslau, N., Davis G.C., \& Anreski, P. (1995). Risk factors for PTSD-related traumatic events: A prospective analysis. American Journal of Psychiatry, 152(4), 529-535. doi: 10.1176/ ajp.152.4.529

Bride, E. (2007). Prevalence of secondary traumatic stress among social workers. Social Work, 52(1), 63-70. doi:10.1093/sw/52.1.63

Bride, B. E., Robinson, M. M., Yegidis, B., \& Figley, C.R. (2004). Development and validation of the secondary traumatic stress scale. Research on Social Work Practice, 14(1), 27-35. doi: 10.1177/10497315025416

Carvajal, C. (2002). Trastorno por estrés postraumático: Aspectos clínicos. Revista Chilena de NeuroPsiquiatría, 40, 20-34. doi:10.4067/S071792272002000600003
Chrestman, K. (1995). Secondary exposure to trauma and self-reported distress among therapists. En B. H. Stamm (Ed.), Secondary traumatic stress: Self-care issues for clinicians, researchers, \& educators ( $2^{\text {nd. }}$ ed., pp. 29-36). Lutherville, MD: Sidran Press.

Cieslak, R., Shoji, K., Douglas A., Melville E., Luszczynsky A., \& Benight C.C. (2014). A metaanalysis of the relationship between job burnout and secondary traumatic stress among workers with indirect exposure to trauma. Psychological Services, 11(1), 75-86. doi: 10.1037/a0033798

Cuffe, S., Addy, C., Garrison, C., Waller, J. L., Jackson, K. L., McKeown, R. E., \& Chilappagari, S. (1998). Prevalence of PTSD in a community sample of older adolescents. Journal of the American Academy of Child and Adolescent Psychiatry, 37(2), 147-154. doi: 10.1097/00004583199802000-00006

Dunn, E., Nishimi, K., Powers, A., \& Bradley, A. (2017). Is developmental timing of trauma exposure associated with depressive and posttraumatic stress disorder symptoms in adulthood? Journal of Psychiatric Research, 84(13) 119-127. doi: 10.1016/j.jpsychires.2016.09.004

Figley, C. R. (1995). Compassion fatigue: Coping with secondary traumatic stress disorder in those who treat the traumatized. New York: BrunnerRoutledge.

Figley, C. R. (1999). Compassion fatigue: Toward a new understanding of the costs of caring. En B. H. Stamm (Ed.), Secondary traumatic stress: Self-care issues for clinicians, researchers, \& educators ( $2^{\text {nd. }}$ ed., pp. 3-28). Lutherville, MD: Sidran Press.

Follette, V., Polusny, M., \& Milbeck, K. (1994). Mental health and law enforcement professionals: Trauma history, psychological symptoms, and impact of providing services to child sexual abuse survivors. Professional Psychology: Research and Practice, 25(3), 275-282. doi: 10.1037/0735-7028.25.3.275

Gibbons, S., Murphy, D., \& Joseph, S. (2011). Countertransference and positive growth in social workers. Journal of Social Work Practice: PsychotherapeuticApproaches in Health, Welfare and the Community, 25(1), 17-30. doi:10.1080/02650530903579246 
Glas, A. S., Lijmer, J. G., Prins, M. H., Bonsel, G. J., \& Bossuyt, P. M. (2003). The diagnostic odds ratio: A single indicator of test performance. Journal of Clinical Epidemiology, 56(2003), 1129-1135. doi: 10.1016/S0895-4356(03)00177-X

Horesh, D. (2015). The reconstruction of criterion A in DSM-5: Is it a true incorporation of secondary traumatization into the PTSD diagnosis? Journal of Loss and Trauma, 21(5), 345-349. doi: 10.1080/15325024.2015.1072016

Katsavouni, F., Bebetsos, E., Malliou, P., \& Beneka, A. (2016). The relationship between burnout, PTSD symptoms and injuries in firefighters. Occupational Medicine, 66(1), 32-37. doi: 10.1093/occmed/kqv144

Lima, E. de P., \& Assunção, A. Á. (2011). Prevalência e fatores associados ao Transtorno de Estresse Pós-Traumático (TEPT) em profssionais de emergência: Uma revisão sistemática da literatura. Revista Brasileira de Epidemiologia, 14(2), 217230. doi: 10.1590/S1415-790X2011000200004

Loo, G. T., DiMaggio, C. J., Gershon, R. R., Canton, D. B., Morse, S. S., \& Galea, S. (2016). Coping behavior and risk of post-traumatic stress disorder among federal disaster responders. Disaster Medicine and Public Health Preparedness, 10(1), 108-117. doi: 10.1017/dmp.2015.141

McCann, L., \& Pearlman, L. (1990). Vicarious traumatization: A framework for understanding the psychological effects of working with victims. Journal of Traumatic Stress, 3, 131-149. doi: 10.1007/BF00975140
McElroy, L., \& McElroy, R. (1991). Countertransference issues in the treatment of incest families. Psychotherapy Theory Research \& Practice, 28(1), 48-54. doi: 10.1037/0033-3204.28.1.48

Meadors, P., Lamson, A., Swanson, M., White, M., \& Sira, N. (2010). Secondary traumatization in pediatric healthcare providers: Compassion fatigue, burnout, and secondary traumatic stress. Omega Journal of Death and Dying, 60(2), 103128. doi: 10.2190/OM.60.2.a

Prati, G., \& Pietrantoni, L. (2009). Optimism, social support, and coping strategies as factors contributing to posttraumatic growth: A metaanalysis. Journal of Loss and Trauma, 14(5), 364-388. doi: 10.1080/15325020902724271

Radke-Yarrow, M., \& Sherman, T. (1992). Hard growing: children who survive. En J. Rolf, A. S. Masten, D. Cicchetti, K. H. Nuechterlein, \& S. Weintraub (Eds.). Risk and protective factors in the development of psychopathology (pp. 97-119). Cambridge: Cambridge University Press.

Stamm, B. H. (2010). The concise ProQOL Manual (2 $2^{\text {nd }}$ ed.). Pocatello: ProQOL.org.

Wrenn, L. J. (2005). The relationship between personal trauma exposure and secondary traumatic stress for social workers. Dissertation Abstracts International, 66(05), 1963A. 